\title{
Simulation of the hyperfine-resolved Zeeman spectrum of Eu atoms in a magnetic trap
}

\section{Citation}

Cai, Long, Bretislav Friedrich, and John M. Doyle. 2000. “Simulation of the Hyperfine-Resolved Zeeman Spectrum of Eu Atoms in a Magnetic Trap." Physical Review A 61 (3) (February 15). doi:10.1103/physreva.61.033412.

\section{Published Version}

doi:10.1103/physreva.61.033412

\section{Permanent link}

http://nrs.harvard.edu/urn-3:HUL.InstRepos:28422147

\section{Terms of Use}

This article was downloaded from Harvard University's DASH repository, and is made available under the terms and conditions applicable to Other Posted Material, as set forth at http:// nrs.harvard.edu/urn-3:HUL.InstRepos:dash.current.terms-of-use\#LAA

\section{Share Your Story}

The Harvard community has made this article openly available.

Please share how this access benefits you. Submit a story.

\section{Accessibility}




\title{
Simulation of the hyperfine-resolved Zeeman spectrum of Eu atoms in a magnetic trap
}

\author{
Long Cai, ${ }^{*}$ Bretislav Friedrich, ${ }^{*}$ and John M. Doyle \\ Department of Physics, Harvard University, Cambridge, Massachusetts 02138
}

(Received 29 September 1999; published 15 February 2000)

\begin{abstract}
We present a simulation of the laser absorption spectra of Eu atoms in the $a^{8} S_{7 / 2}-y^{8} P_{7 / 2}$ band at $462.7 \mathrm{~nm}$ measured previously in a buffer-gas-loaded magnetic trap [J. Kim et al., Phys. Rev. Lett. 78, 3665 (1997)]. The simulations of the hyperfine-resolved Zeeman spectra are based on exact magnetic eigenproperties of the states involved, and yield a complete assignment of all the features observed. This reveals that apart from the $M_{J}$ $=7 / 2$ state and its hyperfine substates, the $M_{J}=5 / 2$ and $3 / 2$ states are also trapped at $B_{\max }=0.52 \mathrm{~T}$ and a temperature of about $250 \mathrm{mK}$.
\end{abstract}

PACS number(s): 32.80.Pj, 32.30.Jc, 39.30.+w, 06.30.Ft

\section{INTRODUCTION}

In a recent experiment, we trapped up to $10^{12}$ ground state $\mathrm{Eu}$ atoms at a temperature close to $250 \mathrm{mK}$ in a buffer-gasloaded magnetic trap [1]. The Eu atoms were detected by laser absorption spectroscopy with a resolution sufficient to distinguish many of the hyperfine Zeeman transitions. The assignment of the spectra was based on a simple model calculation which made use of the analytic magnetic eigenproperties of the $\mathrm{Eu}$ atoms in the strong-field limit. Here we present a simulation of the Eu trap spectra based on exact eigenproperties. This simulation provides an assignment of all the observed features in the trap spectrum and yields an improved fit to the data.

The principle of buffer-gas loading and a detailed description of our apparatus are given in our previous papers [1-3]. Briefly, our magnetic trap is a linear quadrupole field formed by two superconducting coils arranged in an anti-Helmholtz configuration. In the center between the two coils is a zero field point from which the magnitude of the field increases linearly over the trapping region in any direction. This configuration confines atoms that are in the low-field-seeking states (i.e., states whose energy increases with increasing field strength; as a result, they seek regions of minimum field strength where their energy is lowest). For a magnetic dipole moment of $7 \mu_{B}$ (Bohr magneton), corresponding to the $M_{J}$ $=7 / 2$ state of $\operatorname{Eu}\left(a^{8} S_{7 / 2}\right)$, and a magnetic field strength at the trap edge $B_{\text {max }} \approx 0.52 \mathrm{~T}$, the maximum depth of the magnetic trap is $2.4 \mathrm{~K}$. Eu atoms are produced and trapped within a copper cell located in the bore of the magnet. The cell is filled with ${ }^{3} \mathrm{He}$ buffer gas and its temperature is maintained by thermal contact with a dilution refrigerator. The bottom of the cell is outfitted with a fused silica window to enable optical access. The Eu atoms are created by ablating a solid sample of Eu metal $\left(99.9 \%\right.$ pure, with the ${ }^{151} \mathrm{Eu}$ and ${ }^{153} \mathrm{Eu}$ isotopes in their natural abundances) placed within the cell at the edge of the trapping region with a $10 \mathrm{~mJ}, 7 \mathrm{~ns}$ pulse of a frequency doubled yttrium aluminum garnet (YAG) laser. The trapping procedure starts by raising the temperature of the cell to about $250 \mathrm{mK}$ by a resistive heater (resulting in a ${ }^{3} \mathrm{He}$ number density of about $10^{16} \mathrm{~cm}^{-3}$ ) and

\footnotetext{
*Also at Department of Chemistry and Chemical Biology, Harvard University, Cambridge, MA 02138.
}

firing the ablation laser (at which point the heater is turned off). The initially hot $\mathrm{Eu}$ atoms diffuse through the helium gas and quickly thermalize with it via elastic collisions [1]. The cell temperature first rises over about $1 \mathrm{~s}$ due to heating by the ablation pulse but then drops to roughly $170 \mathrm{mK}$ over 20 s. The corresponding ${ }^{3} \mathrm{He}$ number density decreases to about $4 \times 10^{13} \mathrm{~cm}^{-3}$ as a result of which the trapped $\mathrm{Eu}$ ensemble thermally uncouples from the buffer gas and from the cell.

The trapped Eu atoms are detected by laser absorption spectroscopy in the $a^{8} S_{7 / 2}-y^{8} P_{7 / 2}$ band at $462.7 \mathrm{~nm}(6 \mathrm{~ns}$ lifetime [5]). A probe beam is produced using a $\mathrm{KNbO}_{3}$ crystal to double the output of an actively stabilized Ti:sapphire laser. The typical (doubled) power used to probe the atoms is $0.1 \mu \mathrm{W}$. The $3.5 \pm 1.5 \mathrm{~mm}$ diameter beam enters at an angle of about $2^{\circ}$ with respect to the cell axis and passes through the cell center with an offset of $3 \pm 1.5 \mathrm{~mm}$. The beam then reflects from a mirror at the top of the cell, passes the cell center again, exits the cell, and is detected by a photomultiplier tube. The laser is scanned over the entire absorption band of about $13 \mathrm{GHz}$ at a rate of about $15 \mathrm{GHz} / \mathrm{s}$; this allows for ample data averaging at nearly fixed delay times with respect to the ablation pulse.

\section{EXACT HYPERFINE ZEEMAN EIGENPROPERTIES}

The total Hamiltonian $H$ of an atom with total electronic angular momentum $\mathbf{J}$ and nuclear spin $\mathbf{I}$ subject to a magnetic field $\mathbf{B}$ takes the form [4]

$$
H=H_{0}+H_{Z}
$$

with

$$
H_{0}=a \mathbf{I} \cdot \mathbf{J}+b \frac{\frac{3}{2} \mathbf{I} \cdot \mathbf{J}(2 \mathbf{I} \cdot \mathbf{J}+1)-\mathbf{I}^{2} \cdot \mathbf{J}^{2}}{2 I(2 I-1) J(2 J-1)}
$$

the field-free Hamiltonian, and

$$
H_{Z}=g_{J} \mu_{B} \mathbf{J} \cdot \mathbf{B}+g_{I} \mu_{B} \mathbf{I} \cdot \mathbf{B}
$$

the Zeeman Hamiltonian. In the above, $J$ and $I$ are the electronic and nuclear angular momentum quantum numbers, $g_{J}$ and $g_{I}$ the corresponding $g$ factors (see Appendix), $a$ and $b$ the hyperfine magnetic dipole and electric quadrupole coupling constants, and $\mu_{B}$ the Bohr magneton. The magnetic 
TABLE I. Hyperfine constants (in MHz) of selected states of $\mathrm{Eu}$, their $\mathrm{g}$ factors and isotopic shifts (in $\mathrm{MHz}$ ). The natural abundances of the 151 and 153 isotopes are $47.8 \%$ and $52.2 \%$ and both isotopes have a nuclear spin of 5/2. Constants taken from Ref. [6].

\begin{tabular}{lccrrrr}
\hline \hline State & $g_{J}$ & ${ }^{151} a$ & ${ }^{153} a$ & ${ }^{151} b$ & ${ }^{153} b$ & Isotopic shift \\
\hline$a^{8} S_{7 / 2}^{u}$ & 1.99 & -20.05 & -8.85 & -0.7 & -1.78 & \\
\hline$y^{8} P_{7 / 2}^{g}$ & 1.929 & -219.1 & -97 & -295 & -753 & -2977 \\
\hline \hline
\end{tabular}

field has a magnitude $B \equiv|\mathbf{B}|$ and its direction determines the space-fixed $Z$ axis. We neglect higher-order interactions (such as the magnetic octupole interaction).

Generally, either the coupled $|F, M\rangle$ or the uncoupled $\left|M_{J}, M_{I}\right\rangle$ basis can be used to represent the Hamiltonian matrix. Since neither basis function is an eigenfunction of $H$, the Hamiltonian matrix is not diagonal. In what follows we use the uncoupled basis where the matrix elements of the Hamiltonian take a somewhat simpler form [4]. The matrix elements $\left\langle M_{J}^{\prime}, M_{I}^{\prime}|H| M_{J}, M_{I}\right\rangle$ are listed for convenience in the Appendix. The wave functions in the uncoupled basis take the form

$$
\Psi_{\tilde{M}_{J}, \tilde{M}_{I}}(B)=\sum_{M_{J}, M_{I}} a_{M_{J}, M_{I}}^{\tilde{M}_{J}, \tilde{M}_{I}}(B)\left|M_{J}, M_{I}\right\rangle \equiv\left|\tilde{M}_{J}, \tilde{M}_{I}\right\rangle
$$

where the expansion coefficients $a_{M_{J}, M_{I}}^{\widetilde{M}_{I}}{\widetilde{M_{I}}}_{M_{I}}$ depend for a given state on the field strength $B$. A given state is labeled by the nominal values $\widetilde{M}_{J}$ and $\widetilde{M}_{I}$ of the quantum numbers $M_{J}$ and $M_{I}$ that pertain to that same state but in the highfield limit. In the case of ground state europium atoms (see Table I), $J=7 / 2$ and $I=5 / 2$. This leads to $(2 J+1)(2 I+1)$ $=48$ wave functions and hence a $48 \times 48$ matrix. Diagonalization of this matrix at a given field strength yields the exact hyperfine Zeeman eigenproperties of the atoms.

\section{TRANSITION PROBABILITIES}

The transition amplitude between states $\left|\tilde{M}_{J}^{\prime}, \tilde{M}_{I}^{\prime}\right\rangle$ $\leftarrow\left|\widetilde{M}_{J}, \tilde{M}_{I}\right\rangle$ due to an electric dipole operator $\mathbf{d}_{q}$ is given by

$$
\begin{aligned}
& \mathcal{A}_{q}\left(\widetilde{M}_{J}^{\prime}, \tilde{M}_{I}^{\prime} \leftarrow \widetilde{M}_{J}, \widetilde{M}_{I}\right)=\left\langle\widetilde{M}_{J}^{\prime}, \tilde{M}_{I}^{\prime}\left|\mathbf{d}_{q}\right| \widetilde{M}_{J}, \widetilde{M}_{I}\right\rangle \\
& =\sum_{M_{J}^{\prime}, M_{I}^{\prime}, M_{J}, M_{I}} a_{M_{J}^{\prime}, M_{I}^{\prime}} a_{M_{J}, M_{I}}\left\langle M_{J}^{\prime}, M_{I}^{\prime}\left|\mathbf{d}_{q}\right| M_{J}, M_{I}\right\rangle
\end{aligned}
$$

with

$$
\left\langle M_{J}^{\prime}, M_{I}^{\prime}\left|\mathbf{d}_{q}\right| M_{J}, M_{I}\right\rangle \propto \delta\left(M_{I}^{\prime}, M_{I}\right)\left\langle J, M_{J}, J^{\prime}, M_{J}^{\prime} \mid 1, q\right\rangle,
$$

where $\left\langle J, M_{J}, J^{\prime}, M_{J}^{\prime} ; 1, q\right\rangle$ is a Clebsch-Gordan coefficient. The transition probability (line strength) is

$$
S_{q}=\mathcal{A}_{q}^{2}
$$

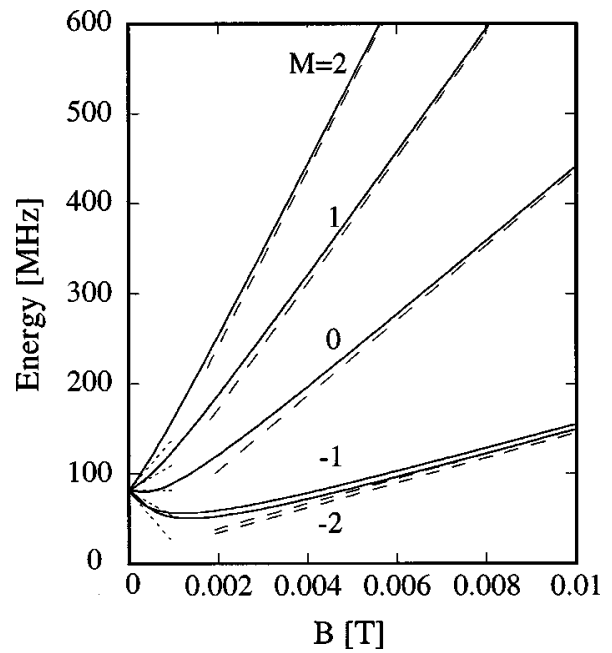

FIG. 1. Comparison of the exact Zeeman eigenenergies with the eigenenergies obtained in the low- and high-field limits for the $F$ $=2$ family of states; see text.

with $q=0$ or \pm 1 for parallel and perpendicular transitions, respectively. The relative weights of the parallel and perpendicular transitions are determined by the angle between the local magnetic field vector and the electric vector of the probe laser beam. For the arrangement used the two types of transitions were found to be nearly equiprobable.

\section{RESULTS AND DISCUSSION}

Figure 1 gives a comparison, for the $F=2$ family of states of ${ }^{153} \mathrm{Eu}$, of the exact eigenenergies with the eigenenergies obtained in the low-and high-field limits, Eqs. (A5) and (A6). One can see that the low-field limit applies only at field strengths of up to about $0.001 \mathrm{~T}$ while the strong-field limit takes over at about $0.005 \mathrm{~T}$. This is in keeping with the nature of the two limiting cases. The low- and high-field limits obtain respectively from the coupled and uncoupled basis representation of $H$ by neglecting the off-diagonal matrix elements. In the uncoupled basis (used here), the offdiagonal elements decrease as $B$ increases, thus allowing the high-field limit to be reached. The magnitudes of the matrix elements at a given field strength reflect the interplay between the magnetic term, proportional to $\mu_{B}$, and the hyperfine constants $a$ and $b$. Since $1 \mu_{B}=14 \mathrm{GHz} / \mathrm{T}$ and both $a$ and $b$ are on the order of $\mathrm{MHz}$, the high-field limit is bound to prevail at $B \approx 10^{-2} \mathrm{~T}$, as observed. The correlation between the low- and high-field limits for either $\operatorname{Eu}\left(a^{8} S_{7 / 2}\right)$ or $\operatorname{Eu}\left(y^{8} P_{7 / 2}\right)$ states is shown in Fig. 2.

Figure 3 shows a profile of the magnetic field strength along the probe beam. The underlying magnetic field strength distribution was calculated from the known parameters of the solenoids. The field distribution provides a basis for the evaluation of the Boltzmann factors of the initial atomic states. The distribution of the number density $n(B)$ of atoms in a low-field-seeking state with eigenenergy $E(B)$ $\geqslant 0$ at the loading temperature $T$ is given by

$$
n(B)=n_{0} \exp \left[-\frac{E(B)}{k T}\right]
$$




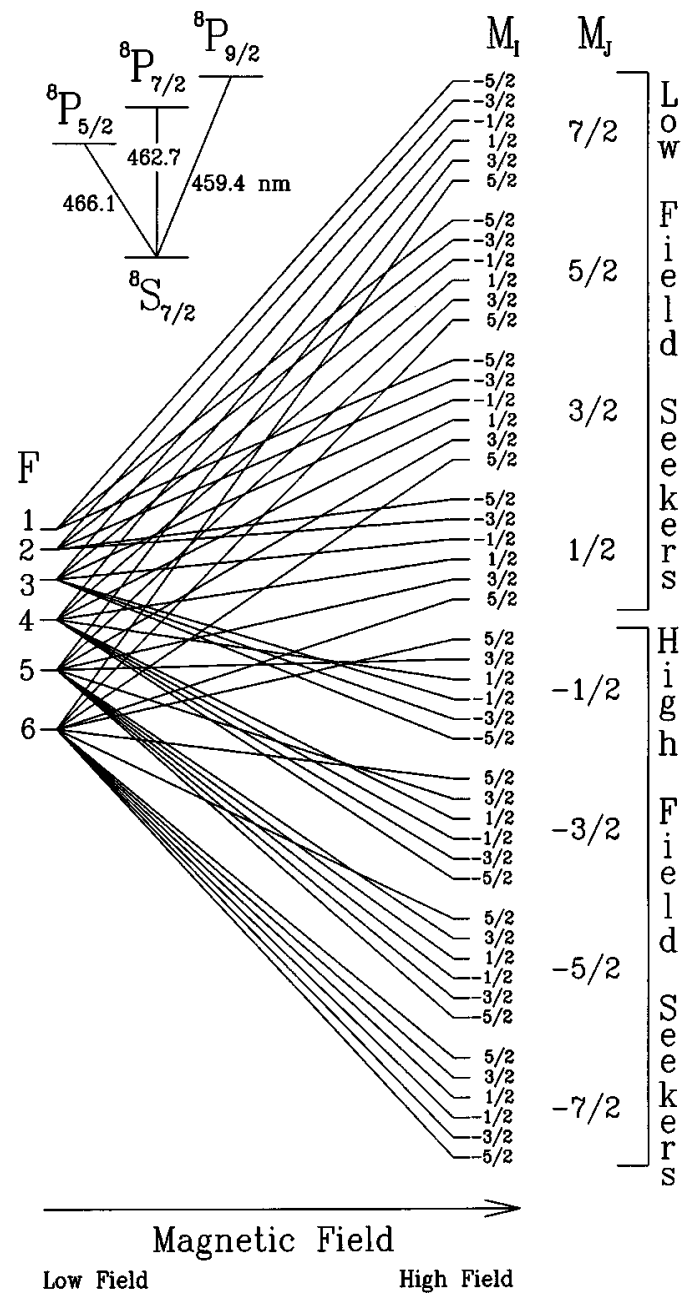

FIG. 2. Correlation diagram between the low- and high-field limits for the states of either of the stable isotopes of $\operatorname{Eu}\left(a^{8} S_{7 / 2}\right)$. Also shown is the transition to the $y^{8} P_{7 / 2}$ state used to probe the atoms.

where $n_{0}$ is the number density at the center of the trap. The differential number $d N(B)$ of atoms exposed to a field strength between $B$ and $B+d B$ is then given by

$$
d N(B)=n(B) d V(B)
$$

with $d V(B)$ the volume element of the trap [3]. Integrating Eq. (8) over the trap volume results in $N=n_{0} V_{\text {eff }}$ where $N$ is the total number of atoms and $V_{\text {eff }}$ is the "effective" volume of the trap. At $B=0$, the hyperfine populations are determined essentially by the degeneracy factors $P(F) \rightarrow(2 F$ $+1)$, i.e., all states $|F, M\rangle$ have the same weight. This was corroborated by our measurements. The intensities, $\mathcal{I}$ of the transitions between states in the absorption spectra are then given by

$$
\mathcal{I}=N S .
$$

Figure 4 shows a sample spectrum of the trapped ensemble of Eu atoms at $0.52 \mathrm{~T}$ trap depth and a loading temperature of $250 \mathrm{mK}$. The spectrum was measured at delay

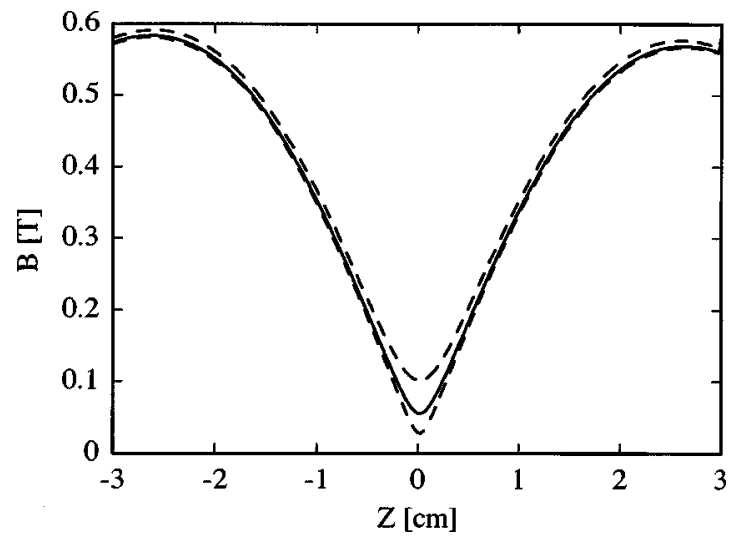

FIG. 3. Profile of the magnetic field strength along the probe beam. The dashed lines correspond to the field at the perimeter of the probe beam.

times with respect to the ablation pulse ranging between 20 and $40 \mathrm{~s}$, i.e., after the ${ }^{3} \mathrm{He}$ buffer gas was cryopumped away.

An essentially identical spectrum was obtained at delay times ranging between 40 and $60 \mathrm{~s}$ after the ablation pulse, just with a poorer signal-to-noise ratio (the atoms were previously determined to leave the trap at an effective two-body loss rate of $\left.(2.5 \pm 1.5) \times 10^{-13} \mathrm{~cm}^{3} / \mathrm{s}[1]\right)$. The best signalto-noise ratio was attained for the spectrum at delay times of up to $20 \mathrm{~s}$ [1]. However, the relative contributions to the structured features in the spectrum from the ${ }^{151} \mathrm{Eu}$ and ${ }^{153} \mathrm{Eu}$ isotopes as well as the linewidths are different from the spec-

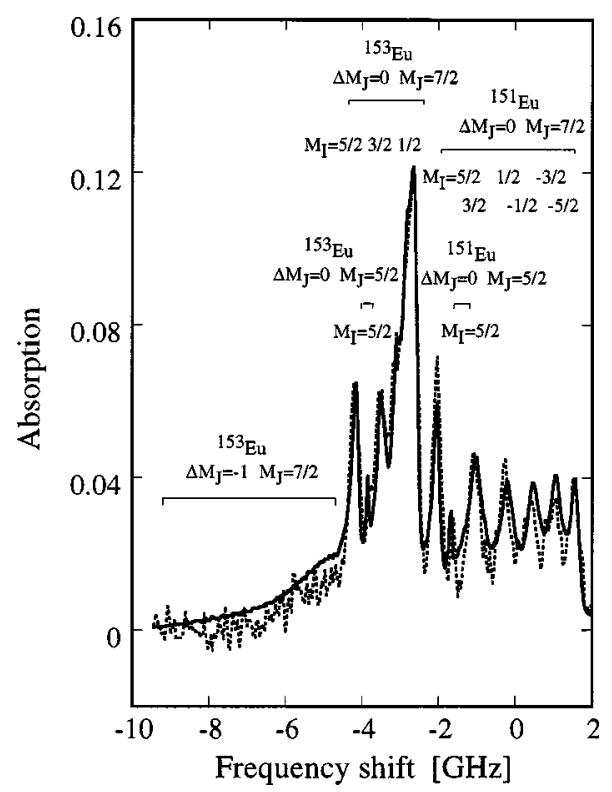

FIG. 4. Sample absorption spectrum of the trapped ensemble of $\mathrm{Eu}$ at $0.52 \mathrm{~T}$ in the $a^{8} S_{7 / 2}-y^{8} P_{7 / 2}$ band at $462.7 \mathrm{~nm}$ measured $40 \mathrm{~s}$ after the ablation pulse (dotted line). The simulated spectrum (full line) provides the indicated assignment of the lines; see text. For each isotope, there are two subbands of magnetic hyperfine transitions, with $\Delta M_{J}=0$ and -1 . Note that for the $\Delta M_{J}=0$ transitions in the ${ }^{151} \mathrm{Eu}$ isotope, all six $M_{I}$ nuclear spin states are clearly resolved. 
tra at $40 \mathrm{~s}$ and $60 \mathrm{~s}$ delay (and from the simulation). We ascribe this difference to the presence of the He buffer gas, although we are unable to identify the mechanism responsible for it.

A diagnostic spectral feature is the plateau region between -10 and $-5 \mathrm{GHz}$, which is mainly composed of the $\Delta M_{J}=-1$ transition from the $M_{J}=7 / 2$ state. Because this transition has a relatively large magnetic broadening, its shape is indicative of the distribution of atoms at different field strengths. The greater the shift, the higher the field to which the atoms are exposed, as seen in the $20 \mathrm{~s}$ spectra of Ref. [1]. In the $40 \mathrm{~s}$ spectra, Fig. 4, the plateau is less frequency-shifted, indicating that the $\mathrm{Eu}$ atoms have cooled and concentrated in the low-field region at the center of the trap.

Figure 5 shows the contributions to the spectrum from the $M_{J}=7 / 2$ (b), 5/2 (c), and 3/2 (d) initial states; (a) provides a summary where the contributions are plotted on the same scale. One can see that most of the spectrum arises from $\operatorname{Eu}\left(M_{J}=7 / 2\right)$. However, there is a sizable contribution from the $M_{J}=5 / 2$ state, which gives rise to a prominent peak at a shift of about $-2 \mathrm{GHz}$ due to a transition from the $M_{I}$ $=5 / 2$ hyperfine state.

\section{CONCLUSIONS}

We simulated the laser absorption spectra of magnetically trapped Eu atoms in the $a^{8} S_{7 / 2}-y^{8} P_{7 / 2}$ band using exact eigenenergies and transition probabilities. These were obtained from a numerical diagonalization of the corresponding $48 \times 48$ Hamiltonian matrix. The simulation yields a complete assignment of all the features observed in our previous experiment and provides an improved fit to the data. Apart from the $M_{J}=7 / 2$ state and its hyperfine substates, the $M_{J}$ $=5 / 2$ and $3 / 2$ states are also found to be trapped at $B_{\max }$ $=0.52 \mathrm{~T}$ and a temperature of about $250 \mathrm{mK}$.

\section{ACKNOWLEDGMENTS}

We thank R. deCarvalho and J. D. Weinstein for helpful discussions. L.C. is grateful for support by the Harvard College Research Program. This material is based upon work supported by the National Science Foundation under Grant No. PHY-9511951.

\section{APPENDIX}

The matrix elements of the Hamiltonian in the uncoupled basis can be found, e.g., in Ref. [4] and are summarized here for convenience. The nonvanishing diagonal elements are

$$
\begin{aligned}
& \left\langle M_{J}, M_{I}\left|H_{0}\right| M_{J}, M_{I}\right\rangle \\
& =a M_{I} M_{J}+\frac{b^{\prime}}{2}\left[3 M_{I}^{2}-I(I+1)\right]\left[3 M_{J}^{2}-J(J+1)\right], \\
& \left\langle M_{J}, M_{I}\left|H_{Z}\right| M_{J}, M_{I}\right\rangle=\mu_{B} B\left(g_{J} M_{J}+g_{I} M_{I}\right),
\end{aligned}
$$

and the nonvanishing nondiagonal elements are

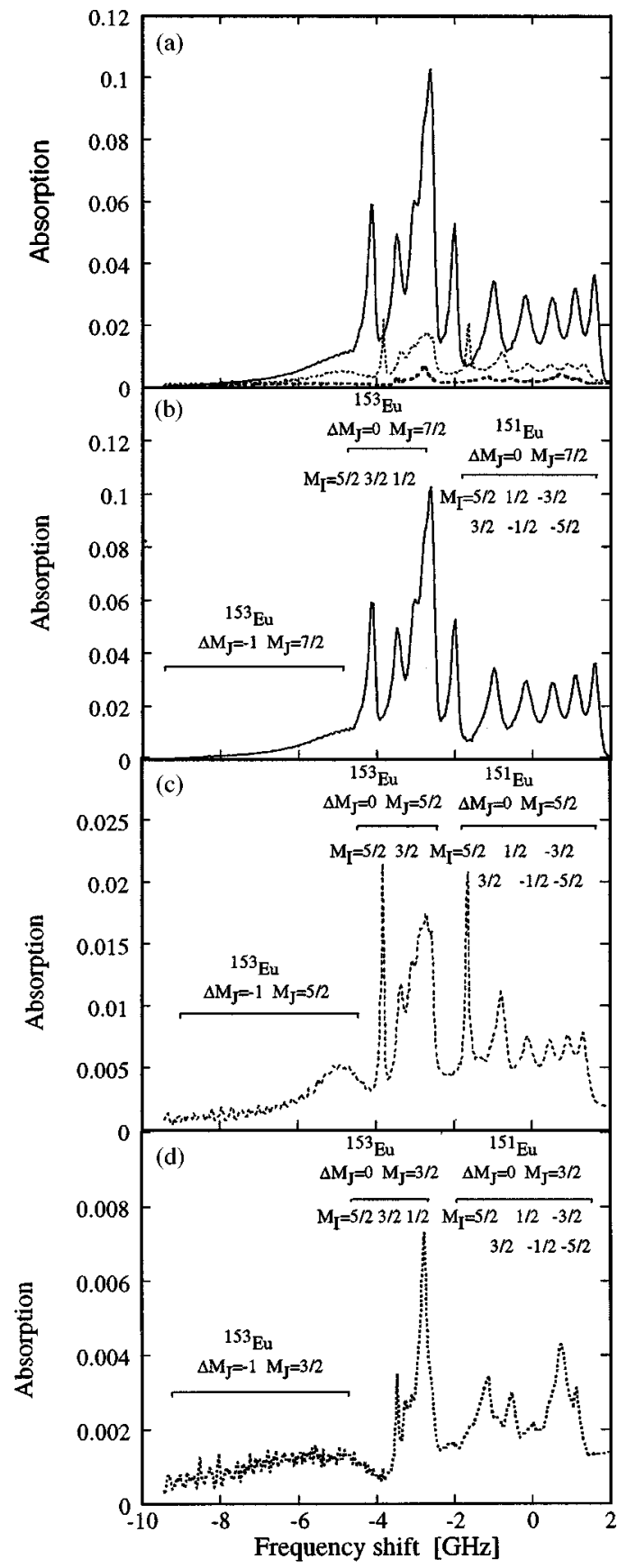

FIG. 5. Relative contributions to the spectrum of Fig. 4 from the initial states with $M_{J}=7 / 2$ (b), $5 / 2$ (c), and $3 / 2$ (d). (a) shows all three contributions on the same scale $\left(M_{J}=7 / 2\right.$ by full line, $5 / 2$ by dashed line, and $3 / 2$ by dotted line).

$$
\begin{aligned}
\left\langle M_{J}, M_{I}\left|H_{0}\right| M_{J} \mp 1, M_{I} \pm 1\right\rangle & \\
= & \frac{1}{2} a\left[J(J+1)-M_{J}\left(M_{J} \mp 1\right)\right]^{1 / 2} \\
& \times\left[I(I+1)-M_{I}\left(M_{I} \pm 1\right)\right]^{1 / 2} \\
& +\frac{3 b^{\prime}}{4}\left(2 M_{J} \mp 1\right)\left(2 M_{I} \pm 1\right) \\
& \times\left[\left(J \pm M_{J}\right)\left(J \mp M_{J}+1\right)\left(I \mp M_{I}\right)\left(I \pm M_{I}+1\right)\right]^{1 / 2},
\end{aligned}
$$




$$
\begin{aligned}
&\left\langle M_{J}, M_{I}\left|H_{0}\right| M_{J} \mp 2, M_{I} \pm 2\right\rangle \\
&= \frac{3 b^{\prime}}{4}\left[\left(J \pm M_{J}\right)\left(J \pm M_{J}-1\right)\left(J \mp M_{J}+1\right)\left(J \mp M_{J}+2\right)\right. \\
&\left.\times\left(I \mp M_{I}\right)\left(I \mp M_{I}-1\right)\right]^{1 / 2} \\
& \times\left[\left(I \pm M_{I}+1\right)\left(I \pm M_{I}+2\right)\right]^{1 / 2}
\end{aligned}
$$

with

$$
b^{\prime} \equiv \frac{b}{2 I(2 I-1) J(2 J-1)} .
$$

The transformation between the coupled and uncoupled basis sets is given by the Clebsch-Gordan coefficients $\left\langle J M_{J}, I M_{I} \mid F M\right\rangle$,

$$
|F, M\rangle=\sum_{M_{J}, M_{I}}\left\langle J M_{J}, I M_{I} \mid F M\right\rangle\left|M_{J}, M_{I}\right\rangle .
$$

The Hamiltonian matrix consists of 13 block-diagonal matrices whose dimensions range between 1 and 6 . Each block pertains to a given value of the good quantum number $M$ $=M_{J}+M_{I}$.

\section{Low-field limit}

In the low-field limit, the angular momenta $\mathbf{J}$ and $\mathbf{I}$ couple to a resultant total angular momentum $\mathbf{F}=\mathbf{J}+\mathbf{I}$ characterized by a quantum number $F=J+I, J+I-1, \ldots,|J-I|$; each value of $F$ has $2 F+1$ projections $M=-F,-F+1, \ldots$, $+F$ on the space-fixed axis defined by the direction of $\mathbf{B}$. Note that there are $\Sigma_{F}(2 F+1)=(2 J+1)(2 I+1)$ coupled states $|F, M\rangle$. The eigenenergy is given by [4]

$$
\begin{aligned}
E(F, M)= & \frac{1}{2} a K+b \frac{\frac{3}{4} K(K+1)-I(I+1) J(J+1)}{2 I(2 I-1) J(2 J-1)} \\
& +M g_{F} \mu_{B} B,
\end{aligned}
$$

with

$$
K \equiv F(F+1)-J(J+1)-I(I+1) .
$$

\section{Strong-field limit}

In the strong-field limit, the angular momenta $\mathbf{J}$ and $\mathbf{I}$ are no longer integrals of motion but their projections $M_{J}$ and $M_{I}$ on the space-fixed axis are. Since $M_{J}$ and $M_{I}$ take respectively $(2 J+1)$ and $(2 I+1)$ values, there are $(2 J+1)$ $\times(2 I+1)$ uncoupled states $\left|M_{J}, M_{I}\right\rangle$. In the uncoupled basis, the eigenvalues of Hamiltonian (1) are given by [4]

$$
\begin{aligned}
E\left(M_{J}, M_{I}\right)= & M_{J} g_{J} \mu_{B} B+M_{I} g_{I} \mu_{B} B+a M_{J} M_{I} \\
& +\frac{9 b}{4 I(2 I-1) J(2 J-1)}\left[M_{J}^{2}-\frac{1}{3} J(J+1)\right] \\
& \times\left[M_{I}^{2}-\frac{1}{3} I(I+1)\right] .
\end{aligned}
$$

The $g$ factors are given by

$$
\begin{aligned}
g_{F}= & g_{J} \frac{F(F+1)+J(J+1)-I(I+1)}{2 F(F+1)} \\
& +g_{I} \frac{F(F+1)+I(I+1)-J(J+1)}{2 F(F+1)}
\end{aligned}
$$

with

$$
\begin{aligned}
g_{J}= & \frac{J(J+1)+L(L+1)-S(S+1)}{2 J(J+1)} \\
& +g_{S} \frac{J(J+1)+S(S+1)-L(L+1)}{2 J(J+1)},
\end{aligned}
$$

$S$ and $L$ the total electronic spin and orbital angular momentum quantum numbers, and $g_{S}$ the gyromagnetic ratio of the electron.
[1] J. Kim, B. Friedrich, D.P. Katz, D. Patterson, J.D. Weinstein, R. deCarvalho, and J.M. Doyle, Phys. Rev. Lett. 78, 3665 (1997).

[2] J.M. Doyle, B. Friedrich, J. Kim, and D. Patterson, Phys. Rev. A 52, R2515 (1995).

[3] R. deCarvalho, B. Friedrich, J.M. Doyle, J. Kim, D. Patterson, and J.D. Weinstein, Eur. Phys. J. D 7, 289 (1999).

[4] N.F. Ramsey, Molecular Beams (Clarendon Press, Oxford, 1956).

[5] K.B. Blagoev and V.A. Komarovskii, At. Data Nucl. Data Tables 56, 1 (1994).

[6] G.J. Zaal et al., Z. Phys. A 290, 339 (1979). 